\title{
Exact Results for Hydrogen Recombination on Dust Grain Surfaces
}

\author{
Ofer Biham ${ }^{1}$ and Azi Lipshtat ${ }^{1}$ \\ 1 Racah Institute of Physics, The Hebrew University, Jerusalem 91904, Israel
}

\begin{abstract}
The recombination of hydrogen in the interstellar medium, taking place on surfaces of microscopic dust grains, is an essential process in the evolution of chemical complexity in interstellar clouds. Molecular hydrogen plays an important role in absorbing the heat that emerges during gravitational collapse, thus enabling the formation of structure in the universe. The $\mathrm{H}_{2}$ formation process has been studied theoretically, and in recent years also by laboratory experiments. The experimental results were analyzed using a rate equation model. The parameters of the surface, that are relevant to $\mathrm{H}_{2}$ formation, were obtained and used in order to calculate the recombination rate under interstellar conditions. However, it turned out that due to the microscopic size of the dust grains and the low density of $\mathrm{H}$ atoms, the rate equations may not always apply. A master equation approach that provides a good description of the $\mathrm{H}_{2}$ formation process was proposed. It takes into account both the discrete nature of the $\mathrm{H}$ atoms and the fluctuations in the number of atoms on a grain. In this paper we present a comprehensive analysis of the $\mathrm{H}_{2}$ formation process, under steady state conditions, using an exact solution of the master equation. This solution provides an exact result for the hydrogen recombination rate and its dependence on the flux, the surface temperature and the grain size. The results are compared with those obtained from the rate equations. The relevant length scales in the problem are identified and the parameter space is divided into two domains. One domain, characterized by first order kinetics, exhibits high efficiency of $\mathrm{H}_{2}$ formation. In the other domain, characterized by second order kinetics, the efficiency of $\mathrm{H}_{2}$ formation is low. In each of these domains we identify the range of parameters in which, due to the small size of the grains, the rate equations do not account correctly for the recombination rate and the master equation is needed.

PACS numbers: PACS:05.10.-a,82.65.+r,98.58.-w
\end{abstract}




\section{INTRODUCTION}

The recombination of hydrogen on the surfaces of microscopic dust grains in the interstellar medium has attracted much interest in recent years. This process is essential since gas-phase reactions cannot account for the abundance of $\mathrm{H}_{2}$ in interstellar clouds [1, 2, 3, 4]. Theoretical [5, 6, 7, 8, 9, 10, 11, 12] and experimental [13, 14, 15, 16, 17, 18] techniques have been used in order to evaluate the rate of $\mathrm{H}_{2}$ formation on relevant dust materials under interstellar conditions. Quantum mechanical calculations and molecular dynamics simulations were performed, in attempt to identify the diffusion and reaction rates on the surfaces of various astrophysically relevant materials [2, 3, 4, 6, 7, 12, 19, 20]. Experimental results were also obtained for the activation energies of the relevant diffusion and desorption processes on various surfaces [13, 14, 15].

Rate equations are an essential tool in the modeling of chemical reactions in the interstellar medium [21, 22, 23, 24, 25, 26, 27, 28, 29, 30, 31, 32. Chemical models based on the rate equation approach take into account a large number of reactions in the gas phase as well as reactions that take place on the surfaces of dust grains. Rate equations were recently used in order to analyze the results of laboratory experiments on $\mathrm{H}_{2}$ formation on dust-analog surfaces [33. The analysis provided the surface parameters that are essential for the evaluation of the $\mathrm{H}_{2}$ formation rate on dust grains in the interstellar medium. The rate equations used in Ref. [33] describe the diffusion, reaction and desorption processes on the surface. They provide the time evolution of the average densities of atoms and molecules on the surface, while fluctuations are neglected. Such rate equations are expected to provide good results for macroscopic surfaces. However, it turns out that they may be unsuitable for the study of hydrogen recombination in the interstellar medium due to the small grain size and low flux 34, 35, 36, 37, 38. Under these conditions the number of $\mathrm{H}$ atoms on the surface of a grain may be very small and fluctuations are expected to be significant. Attempts to resolve this difficulty included the use of modified rate equations in which parameters are changed to account for the finite grain size [34, 35, 36, 37, 38]. Monte Carlo methods were also used, to simulate the surface diffusion and reaction processes on small grains [39, 40]. The class of Monte Carlo methods that are suitable for such activated processes on the surface, away from thermal equilibrium, are the continuous time or kinetic Monte Carlo techniques [41]. In these simulations, at each time step, the next move is picked with a probability propor- 
tional to its rate. The elapsed time is given, according to the theory of stochastic processes, by the inverse of the sum of the rates of all processes that could have occurred at that time. The kinetic Monte Carlo approach can be directly related to the underlying master equation that describes the time evolution of the probabilities of all the microscopic states of the system [42]. Monte Carlo simulations typically require large computational resources. For example, the calculation of averages for quantities such as the $\mathrm{H}_{2}$ formation rate is done by collecting large amounts of statistical information [39, 40]. To study the chemistry of interstellar clouds one needs a model that couples the gas-phase and grain-surface reactions. It was found that the use of Monte Carlo methods in this context is impractical, while rate equations for surface chemistry cannot account correctly for reaction rates on small grains.

Recently it was shown that the $\mathrm{H}_{2}$ formation process on small grains can be described by a master equation approach [43, 44]. The master equation takes into account both the discrete nature of the $\mathrm{H}$ atoms as well as the fluctuations. Its dynamical variables are the probabilities $P(n)$ that there are $n$ atoms on a grain at time $t$. The time derivatives $\dot{P}(n)$, $n=0,1,2, \ldots$ are expressed in terms of the adsorption, reaction and desorption terms. The master equation provides the time evolution of the probabilities $P(n)$, from which the recombination rate can be calculated. It was used in conjunction with surface parameters obtained experimentally and computationally, to explore the hydrogen recombination and other chemical reactions on small grains under interstellar conditions [43, 44, 45].

In this paper we present a comprehensive analysis of the $\mathrm{H}_{2}$ formation process under steady state conditions as a function of the physical parameters. These parameters include the flux of $\mathrm{H}$ atoms, the grain size and surface temperature. They also include properties of the surface, namely the density of adsorption sites as well as the activation energies for $\mathrm{H}$ diffusion and desorption. The analysis is based on an exact analytical solution of the master equation. In this solution, the steady state distribution $P(n)$ is expressed in terms of two dimensionless quantities, composed of the physical parameters mentioned above. Using this solution we identify the relevant length scales in the problem. The parameter space is then divided into two domains. One domain, characterized by first order kinetics (namely, the $\mathrm{H}_{2}$ formation rate is linearly proportional to the flux of $\mathrm{H}$ atoms), exhibits high efficiency of $\mathrm{H}_{2}$ formation. In the other domain, characterized by second order kinetics (namely, the $\mathrm{H}_{2}$ formation rate is proportional to the square of the incoming flux), the efficiency of $\mathrm{H}_{2}$ formation is low. In each of these domains we identify the range of parameters in which, 
due to the small size of the grains, the rate equations do not apply and the master equation is needed.

The paper is organized as follows. The rate equation model is described in Sec. IIA and analyzed in Sec. IIB. The master equation is introduced in Sec. IIIA. The exact solution of the master equation is given in Sec. IIIB. The analysis of the $\mathrm{H}_{2}$ formation process on small grains, that is based on this solution, is presented in Sec. IIIC. The results are discussed in Sec. IV and summarized in Sec. V.

\section{II. $\mathrm{H}_{2}$ FORMATION ON MACROSCOPIC SURFACES}

\section{A. The Rate Equation Model}

Consider a flux of $\mathrm{H}$ atoms that are irradiated and stick to a macroscopic surface. The atoms perform hops as random walkers on the surface and recombine into $\mathrm{H}_{2}$ molecules when they encounter one another. Let $\rho(t)$ (in monolayers [ML]) be the coverage of $\mathrm{H}$ atoms on the surface at time $t$. Its time dependence is described by the following rate equation:

$$
\frac{d \rho}{d t}=f-W \rho-2 a \rho^{2}
$$

The first term on the right hand side of Eq. (11) represents the flux of $\mathrm{H}$ atoms. The parameter $f$ represents the effective flux of atoms (in units of $\mathrm{ML} s^{-1}$ ), namely, the (temperature dependent) sticking coefficient $\xi(T)$ is absorbed into $f$ [the Langmuir-Hinshelwood (LH) rejection process [46] of atoms deposited on top of already adsorbed atoms is neglected here since it is assumed that the coverage is low]. The second term in Eq. (11) represents the desorption of $\mathrm{H}$ atoms from the surface. The desorption coefficient is

$$
W=\nu \cdot \exp \left(-E_{1} / k_{B} T\right)
$$

where $\nu$ is the attempt rate (standardly taken to be $10^{12} \mathrm{~s}^{-1}$ ), $E_{1}$ is the activation energy barrier for desorption of an $\mathrm{H}$ atom and $T$ is the temperature. The third term in Eq. (11) accounts for the depletion of the $\mathrm{H}$ population on the surface due to recombination into $\mathrm{H}_{2}$ molecules, where

$$
a=\nu \cdot \exp \left(-E_{0} / k_{B} T\right)
$$


is the hopping rate of $\mathrm{H}$ atoms on the surface and $E_{0}$ is the activation energy barrier for hopping. Here we assume that diffusion occurs only by thermal hopping, in agreement with experimental results [33]. We also assume that there is no energy barrier for recombination. The $\mathrm{H}_{2}$ production rate $r\left(\mathrm{ML} \mathrm{s}^{-1}\right)$ is given by

$$
r=a \rho^{2} .
$$

For simplicity we assume here that all the $\mathrm{H}_{2}$ molecules are desorbed from the surface upon formation. Even if on real surfaces some of the molecules may remain on the surface and desorb thermally later, under steady state conditions, at low coverage, this will not affect the recombination rate.

A (more complete) model, based on Eq. (11) was used [33] to analyze the results of temperature programmed desorption (TPD) experiments [13, 14, 15]. The values of $E_{0}$ and $E_{1}$ (as well as two additional parameters) that best fit the experimental results were obtained. The steady state behavior under astrophysically relevant conditions was then studied and the recombination efficiency

$$
\eta=\frac{r}{f / 2}
$$

was calculated in an astrophysically relevant range of flux and temperature. Note that under steady state conditions $\eta$ is limited to the range $0 \leq \eta \leq 1$. It was found that the recombination efficiency is highly temperature dependent. It exhibits a narrow window of

high efficiency along the temperature axis, which slowly shifts to higher temperatures as the flux is increased [33].

\section{B. Analysis and Results}

Consider a macroscopic surface exposed to a constant flux of $\mathrm{H}$ atoms, as described by Eq. (11). Under steady state conditions $d \rho / d t=0$ and the coverage is given by

$$
\rho=\frac{1}{4}\left(\frac{W}{a}\right)\left[-1+\sqrt{1+8 \frac{(f / W)}{(W / a)}}\right] .
$$

The recombination efficiency $\eta=2(a / f) \rho^{2}$ takes the form 


$$
\eta=\frac{1}{8} \frac{(W / a)}{(f / W)}\left[-1+\sqrt{1+8 \frac{(f / W)}{(W / a)}}\right]^{2} .
$$

To obtain a better understanding of the recombination process we will try to identify the length and time scales involved. Consider the ratio $a / W$ between the hopping rate and the desorption rate. This ratio is (on average) the number of hops an $\mathrm{H}$ atom visits before it desorbs (neglecting recombination). It is also (up to a constant multiplicative factor of order unity and a logarithmic correction) the number of sites that the atom visits before it desorbs [47]. We will denote this number by

$$
s_{\text {visit }}=a / W
$$

Consider the ratio $W / f$ between the desorption rate and the flux. Neglecting the recombination term we obtain $W / f=1 / \rho$, namely this is approximately the average number of vacant sites around each $\mathrm{H}$ atom. We denote it by

$$
s_{\text {vacant }}=W / f
$$

The properties of the recombination process can thus be plotted on a two dimensional parameter space, namely $f / W$ vs. $W / a$ [Fig. 1]. The coverage $\rho$ depends on these two parameters, while $\eta$ depends only on the ratio

$$
\tan \theta=\frac{(f / W)}{(W / a)}, \quad 0 \leq \theta \leq \pi / 2 .
$$

The coverage $\rho$ and the recombination efficiency $\eta$ as a function of $\theta$ along the curve

$$
\frac{f}{W}=c\left(\frac{W}{a}\right)^{-1}
$$

where $c=1 / 2$, are shown in Figs. 2(a) and 2(b), respectively. The solid lines in Figs. 2(a) and 2(b) show the results without LH rejection, while the dashed lines show the results for similar conditions but including LH rejection. In these Figures we identify a domain of high coverage and high efficiency for $f / W>W / a$ and a domain of low coverage and low efficiency for $f / W<W / a$, separated by the diagonal line in Fig. 1. We will now analyze the limits, deep in each of these domains, in terms of the length scales associated with $s_{\text {visit }}$ and $s_{\text {vacant }}$ (the length-scales are obtained by dividing $s_{\text {visit }}$ and $s_{\text {vacant }}$ by the density of adsorption sites 
and taking the square root). In the case $f / W \gg W / a$, namely $s_{\text {visit }} \gg s_{\text {vacant }}$, the typical number of sites that an atom visits is much larger than the number of vacant sites around it. Therefore, it is most likely to find a second $\mathrm{H}$ atom and recombine. In the opposite limit $f / W \ll W / a$, namely $s_{\text {visit }} \ll s_{\text {vacant }}$, most atoms visit only vacant sites around their initial adsorption site and desorb before having a chance to form molecules. As a result, the recombination efficiency $\eta$ is low. Evaluating the coverage in both limits we obtain [48

$$
\rho \cong \begin{cases}\frac{1}{\sqrt{2}} \sqrt{\frac{f}{a}}: & \frac{f}{W} \gg \frac{W}{a} \\ \frac{f}{W}: & \frac{f}{W} \ll \frac{W}{a} .\end{cases}
$$

The $\mathrm{H}_{2}$ production rate $r=a \rho 2$ is given by

$$
r \cong \begin{cases}\frac{1}{2} f: \quad & \frac{f}{W} \gg \frac{W}{a} \\ \frac{a}{W^{2}} f^{2}: & \frac{f}{W} \ll \frac{W}{a} .\end{cases}
$$

In the first limit, $r$ is linear in the flux $f$, thus we denote this limit as first order kinetics. In the second case $r$ is proportional to $f 2$ and we thus denote it as second order kinetics. The regime of first order kinetics is characterized by high recombination efficiency $\eta \cong 1$, since the desorption of $\mathrm{H}$ atoms is negligible. In the second order kinetics, desorption of $\mathrm{H}$ atoms (before they form molecules) is the dominant process. Therefore, the efficiency $\eta$ is low. More precisely, the recombination efficiency is

$$
\eta \cong \begin{cases}1: & \frac{f}{W} \gg \frac{W}{a} \\ \frac{2 a}{W^{2}} f: & \frac{f}{W} \ll \frac{W}{a} .\end{cases}
$$

For a given surface it is convenient to plot the coverage and recombination rate as a function of the flux and the surface temperature [33]. The boundary between the first and second order domains is given by

$$
T_{\text {up }}(f)=\frac{2 E_{1}-E_{0}}{k_{B}(\ln \nu-\ln f)},
$$

with the first order domain for temperatures below this curve and the second order domain above it. For parameters on this boundary line the recombination efficiency is $\eta=1 / 2$.

Note that in Eq. (11) the coverage $\rho$ is not limited to the range $0 \leq \rho \leq 1$. In the LH kinetics, the coverage is bounded from above due to the rejection of atoms deposited on top of other $\mathrm{H}$ atoms that are already adsorbed. To include this effect, the flux term $f$ in Eq. (11) is replaced by $f(1-\rho)$. Solving the new equation at steady state we obtain 


$$
\eta=\frac{1}{8} \frac{(W+f) / a}{f /(W+f)}\left[-1+\sqrt{1+8 \frac{f /(W+f)}{(W+f) / a}}\right]^{2},
$$

which is similar to Eq. (7) except that now $W$ is replaced by $(W+f)$. The LH rejection introduces further constraints on the domain of high efficiency of $\mathrm{H}_{2}$ formation. This is due to the fact that at low temperature the coverage approaches unity and newly deposited atoms are rejected. For simplicity we will define the high efficiency domain as the set of points in parameter space for which $\eta \geq 1 / 2$. The boundary of this domain, namely the curve in the $(W / a, f / W)$ plane on which $\eta=1 / 2$, is given by

$$
W / a=\frac{f / W}{(1+f / W)^{2}} .
$$

In Fig. 3 we show the high efficiency domain (gray area) and the low efficiency domain in parameter space for the LH kinetics. For $f / W \ll 1$ the boundary between them coincides with the diagonal line that separates the first and second order domain without the LH rejection. For any given value of $W / a$ in the range $0<W / a<1 / 4$, the high efficiency domain is bounded from above and below by $f_{\text {low }}(W / a) / W<f / W<f_{\text {up }}(W / a) / W$ where $f_{\text {up }}(W / a)$ and $f_{\text {low }}(W / a)$ are determined by Eq. (17). The two boundaries are related to each other according to

$$
\frac{f_{\text {up }}(W / a)}{W}=\frac{W}{f_{\text {low }}(W / a)} .
$$

For a given surface, for which $E_{0}, E_{1}$ and $\nu$ are known, we can draw a diagram for the

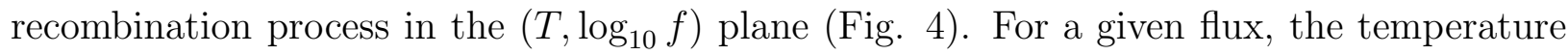
in the domain of high efficiency (gray area) is now bounded from above by Eq. (15) and from below by

$$
T_{\text {low }}(f)=\frac{E_{0}}{k_{B}(\ln \nu-\ln f)} .
$$

Therefore, in order for a given surface to exhibit a domain of high efficiency, the condition $T_{\text {up }}(f)>T_{\text {low }}(f)$ must be satisfied. This condition is satisfied if $E_{1}>E_{0}$, namely the activation energy for desorption is higher than for diffusion.

A further constraint on the high efficiency domain may appear due to the existence of $\mathrm{H}_{2}$ molecules on the surface. These molecules may reject some of the deposited atoms through 
the LH mechanism and thus reduce the effective flux. The $\mathrm{H}_{2}$ molecules on the surface may be either molecules that formed on the surface and did not desorb upon formation [33], or ones that were adsorbed from the gas phase. In both cases the condition for the existence of a high efficiency domain is that $E_{2}<2 E_{1}-E_{0}$, where $E_{2}$ is the activation energy for desorption of $\mathrm{H}_{2}$ molecules from the surface. Thus, surfaces that do not satisfy this condition (particularly surfaces on which $\mathrm{H}_{2}$ molecules are adsorbed more strongly thatn $\mathrm{H}$ atoms) are not expected to efficiently catalyze the $\mathrm{H}_{2}$ formation process, unless molecules are desorbed upon formation and their density in the gas phase is too low to saturate the adsorption sites on the surface.

For small grains and low flux one may reach the situation in which the average number of $\mathrm{H}$ atoms on a grain is of order unity or even less. Under these conditions the rate equation model, that takes into account only average densities, ignoring the fact that $\mathrm{H}$ atoms are discrete entities, does not account correctly for the recombination rate. This is due to the fact that the recombination process requires at least two atoms on the surface and the fluctuations in the number of $\mathrm{H}$ atoms on different grains become dominant. A more complete description of the recombination process is needed. Such description is provided by the master equation presented below.

\section{III. $\mathrm{H}_{2}$ FORMATION ON SMALL DUST GRAINS}

\section{A. The Master Equation Model}

We will now consider the formation of $\mathrm{H}_{2}$ molecules on small dust grains. In this case it is more convenient to rescale the parameters such that instead of using quantities per unit area - the total amount per grain will be used. The number of $\mathrm{H}$ atoms on the grain is denoted by $n$. Its expectation value is given by $\langle n\rangle=S \cdot \rho$ where $S$ is the number of adsorption sites on the grain. The incoming flux of $\mathrm{H}$ atoms onto the grain surface is given by $F=S \cdot f$ (atoms $\mathrm{s}^{-1}$ ). The desorption rate $W$ remains unchanged. The hopping rate $a$ (hops $\mathrm{s}^{-1}$ ) is replaced by $A=a / S$ which is approximately the inverse of the time $t_{s}$ required for an atom to visit nearly all the adsorption sites on the grain surface. This is due to the fact that in two dimensions the number of distinct sites visited by a random walker is linearly proportional to the number of steps, up to a logarithmic correction [47. The $\mathrm{H}_{2}$ production 
rate of a single grain is given by $R=S \cdot r\left(\right.$ molecules $\left.\mathrm{s}^{-1}\right)$. The rate equation will now take the form

$$
\frac{d\langle n\rangle}{d t}=F-W\langle n\rangle-2 A\langle n\rangle^{2}
$$

Under given flux and surface temperature, for grains that are large enough to hold many $\mathrm{H}$ atoms, Eq. (20) provides a good description of the recombination process. However, for small enough grains $\langle n\rangle$ becomes or order unity and Eq. (20) becomes unsuitable, because it neglects the fluctuations in the number of atoms on a grain.

We will now introduce the master equation, that provides a correct description of the recombination process even in the limit of small grain sizes and low flux. Consider a grain that is exposed to a flux $F$ of $\mathrm{H}$ atoms. At any given time the number of $\mathrm{H}$ atoms adsorbed on the grain may be $n=0,1,2, \ldots, S$. The probability that there are $n$ hydrogen atoms on the grain is given by $P(n)$, where

$$
\sum_{n=0}^{S} P(n)=1 .
$$

The master equation provides the time derivatives of these probabilities, $\dot{P}(n)$, namely the gain or loss of the probabilities of the different states. These derivatives are linear in the probabilities themselves. The equations include three terms. The first term describes the effect of the incoming flux $F$. The probability $P(n)$ increases when an $\mathrm{H}$ atom is adsorbed on a grain that already has $n-1$ adsorbed atoms [at a rate of $F P(n-1)$ ], and decreases when a new atom is adsorbed on a grain with $n$ atoms on it [at a rate of $F P(n)$ ]. The adsorption process is considered as completely random (Poisson process), and is fully characterized by $F$. The second term includes the effect of desorption. An $\mathrm{H}$ atom desorbed from a grain with $n$ adsorbed atoms, decreases the probability $P(n)$ [at a rate of $n W P(n)$, where the factor $n$ is due to the fact that each of the $n$ atoms can desorb], and increases the probability $P(n-1)$ at the same rate. The third term describes the effect of recombination. The production of one molecule reduces the number of adsorbed atoms from $n$ to $n-2$. For a given pair of $\mathrm{H}$ atoms, the recombination rate is proportional to the sweeping rate $A$ multiplied by 2 since both atoms are mobile simultaneously. This rate is multiplied by the number of possible pairs of atoms, namely $n(n-1) / 2$. The master equation exhibits the Markov property, namely, no memory effects are included 42]. This property emerges from 
the fact that the incoming flux keeps washing out any spatial correlations that may develop due to recombination events between adjacent atoms. The master equation thus takes the form:

$$
\begin{aligned}
\dot{P}(0) & =-F P(0)+W P(1)+2 \cdot 1 \cdot A P(2) \\
\dot{P}(1) & =F[P(0)-P(1)]+W[2 P(2)-P(1)]+3 \cdot 2 \cdot A P(3) \\
\dot{P}(2) & =F[P(1)-P(2)]+W[3 P(3)-2 P(2)] \\
& +A[4 \cdot 3 \cdot P(4)-2 \cdot 1 \cdot P(2)] \\
& \vdots \\
\dot{P}(n) & =F[P(n-1)-P(n)]+W[(n+1) P(n+1)-n P(n)] \\
& +A[(n+2)(n+1) P(n+2)-n(n-1) P(n)] \\
& \vdots \\
\dot{P}(S) & =F[P(S-1)-P(S)]-S W P(S)-S(S-1) A P(S) .
\end{aligned}
$$

Note that the equations for $\dot{P}(0)$ and $\dot{P}(1)$ do not include all the terms, because at least one $\mathrm{H}$ atom is required for desorption to occur and at least two for recombination. Similarly, the equation for $\dot{P}(S)$ does not include all the terms since there is no room for more than $S$ atoms on the grain surface. The expectation value for the number of $\mathrm{H}$ atoms on the grain is

$$
\langle n\rangle=\sum_{n=0}^{S} n P(n) .
$$

The rate of formation of $\mathrm{H}_{2}$ molecules, $R$ (molecules $\mathrm{s}^{-1}$ ), is thus given by

$$
R=A \sum_{n=2}^{S} n(n-1) P(n) .
$$

The hydrogen recombination efficiency on the grains is given by

$$
\eta=\frac{R}{(F / 2)}
$$




\section{B. Exact Solution of the Master Equation}

When a grain is maintained at a constant temperature (namely $W$ and $A$ are fixed), and is exposed to a constant flux $F$, the recombination process on its surface approaches a steady state. Under steady state conditions the time derivatives on the left hand side of Eq. (22) is zero. We thus obtain a homogeneous set of coupled linear equations in the variables $P(n)$, $n=0,1,2, \ldots, S$. This set can be expressed in a matrix form as

$$
M \vec{P}=\overrightarrow{0},
$$

where

$$
M=\left(\begin{array}{cccccc}
-F & W & 2 A & 0 & \ldots & 0 \\
F & -F-W & 2 W & 3 \cdot 2 A & \ldots & 0 \\
0 & F & -F-2(W+A) & 3 W & \ldots & S \cdot(S-1) A \\
0 & 0 & F & -F-3(W+2 A) & \ldots & S W \\
\vdots & \vdots & \vdots & \vdots & & \vdots \\
0 & 0 & 0 & F & \ldots-F-S[W+(S-1) A]
\end{array}\right)
$$

and $\vec{P}$ consists of the components $P(n), n=0,1, \ldots, S$. The matrix elements are denoted by $M(n, m), n, m=0,1,2, \ldots, S$. The only non-vanishing matrix elements are

$$
\begin{aligned}
& M(n, n)=-[F+n W+n(n-1) A] \\
& M(n+1, n)=F \\
& M(n, n+1)=(n+1) W \\
& M(n, n+2)=(n+2)(n+1) A .
\end{aligned}
$$

For a finite grain the set of equations (22) is truncated by $n \leq S$. However, under interstellar conditions we expect low coverage of $\mathrm{H}$ atoms on the grain, namely $\langle n\rangle \ll S$. Therefore, one can impose a cutoff at some value $s<S$ such that $P(n)=0$ for $n>s$ and the normalization of probabilities now takes the form $\sum_{n=0}^{s} P(n)=1$. The terms in the matrix that represent flow of probabilities between $P(n), n \leq s$ and $P(n), n>s$ are removed. Three of these terms disappear since they are outside the $(s+1) \times(s+1)$ size matrix that 
we now consider. The term $(-F)$ in the matrix element $M(s, s)$, that represents the addition of an $\mathrm{H}$ atom to a grain that already includes $s$ atoms is also removed. The modified term will be $M(s, s)=-s[W+(s-1) A]$.

We proceed by performing linear operations on the rows of the matrix $M$. Starting from the top, we add each element $M(0, m), m=0, \ldots, s$ of the first row to the corresponding element $M(1, m)$ in the second row. We then proceed downwards, adding the elements of the $n$th row to the corresponding elements of the $(n+1)$ th row. Each row of the resulting matrix includes one diagonal term and two off-diagonal terms of the form: $M^{\prime}(n, n)=-F$, $M^{\prime}(n, n+1)=(n+1)(W+n A)$ and $M^{\prime}(n, n+2)=(n+2)(n+1) A$ (except for the last row in which all the elements are zero and the next to the last row in which the second off-diagonal element is removed). In order to remove the second off-diagonal terms $M^{\prime}(n, n+2), n=0, \ldots, s-2$ we now perform a second set of operations, this time starting at the bottom rows. We first subtract from each element $M^{\prime}(s-2, m), m=0, \ldots, s$ of the $(s-2)$ th row, the corresponding element $M^{\prime}(s-1, m)$, of the $(s-1)$ th row, multiplied by $M^{\prime}(s-2, s) / M^{\prime}(s-1, s)$. We then proceed in a similar fashion all the way up. Each line in the resulting matrix has only one diagonal and one off-diagonal term. The diagonal elements take the form $M^{\prime \prime}(n, n)=-F, n=0, \ldots, s-1$ and $M^{\prime \prime}(s, s)=0$. The off-diagonal elements will be

$$
\begin{aligned}
M^{\prime \prime}(s-1, s) & =M^{\prime}(s-1, s) \\
M^{\prime \prime}(s-2, s-1) & =M^{\prime}(s-2, s-1)-\frac{M^{\prime}(s-2, s)}{M^{\prime \prime}(s-1, s)} \cdot M^{\prime}(s-1, s-1) \\
M^{\prime \prime}(s-3, s-2) & =M^{\prime}(s-3, s-2)-\frac{M^{\prime}(s-3, s-1)}{M^{\prime \prime}(s-2, s-1)} \cdot M^{\prime}(s-2, s-2) \\
\vdots & \\
M^{\prime \prime}(s-n, s-n+1) & =M^{\prime}(s-n, s-n+1) \\
& -\frac{M^{\prime}(s-n, s-n+2)}{M^{\prime \prime}(s-n+1, s-n+2)} \cdot M^{\prime}(s-n+1, s-n+1) \\
\vdots & =M^{\prime}(0,1)-\frac{M^{\prime}(0,2)}{M^{\prime \prime}(1,2)} \cdot M^{\prime \prime}(1,1) \\
M^{\prime \prime}(0,1) &
\end{aligned}
$$

By combining the operations in Eq. (29) we express the non-vanishing off-diagonal elements of the matrix $M^{\prime \prime}$ as continued fractions. Their reduced form is 


$$
\frac{M^{\prime \prime}(n, n+1)}{(n+1) \sqrt{A F}}=\sqrt{\frac{A}{F}}\left(\frac{W}{A}+n\right)+\frac{1}{\sqrt{\frac{A}{F}}\left(\frac{W}{A}+n+1\right)+\frac{1}{\cdots+\frac{1}{\sqrt{\frac{A}{F}}\left(\frac{W}{A}+s\right)}}}
$$

for $n=0, \ldots, s-1$. The equation $M^{\prime \prime} \vec{P}=\overrightarrow{0}$ now takes the form of a set of recursion equations:

$$
P(n+1)=\frac{-M^{\prime \prime}(n, n)}{M^{\prime \prime}(n, n+1)} P(n), \quad n=0, \ldots, s-1 .
$$

Using these equations we can express all the probabilities in terms of $P(0)$ according to

$$
P(n)=(-1)^{n} P(0) \prod_{i=0}^{n-1}\left[\frac{M^{\prime \prime}(i, i)}{M^{\prime \prime}(i, i+1)}\right], \quad n=1, \ldots, s .
$$

The probability $P(0)$ is then determined by the normalization condition $\sum_{n=0}^{s} P(n)=1$. Eq. (32), complemented by the normalization condition, provides an exact solution of the master equation under steady state conditions for any finite cutoff $s \leq S$. Since the master equation is of use when the coverage is very low, the tail of $P(n)$ already decays for some $n \ll S$. Therefore, in evaluating $P(0)$ it is sensible to ignore the cutoff at $s \leq S$ and write the infinite sum instead. In this case, the solution of the master equation can be expressed in terms of Bessel functions. The connection to the Bessel functions can be obtained from the (infinite) continued fraction expression in Eq. (30), obtained when $s \rightarrow \infty$. Using the continued fraction expansion of the ratio $J_{\nu}(z) / J_{\nu-1}(z)$ in Ref. 49] (Eq. 9.1.73 in page 363) and the relation $I_{\nu}(z) / I_{\nu-1}(z)=-i J_{\nu}(i z) / J_{\nu-1}(i z)$ we obtain that

$$
\frac{M^{\prime \prime}(n, n+1)}{(n+1) \sqrt{F A}}=\frac{I_{W / A+n-1}(2 \sqrt{F / A})}{I_{W / A+n}(2 \sqrt{F / A})}
$$

We thus obtain an expression for $P(n)$ of the form

$$
P(n)=\frac{1}{n !} P(0)\left(\sqrt{\frac{F}{A}}\right)^{n} \frac{I_{W / A+n-1}(2 \sqrt{F / A})}{I_{W / A-1}(2 \sqrt{F / A})} .
$$

The normalization factor $P(0)$ can be expressed in terms of Bessel functions, using Ref. 49 (Eq. 9.6.51 in page 377, with $\lambda=\sqrt{2}$ ), as

$$
P(0)=\frac{2^{\frac{1}{2}\left(\frac{W}{A}-1\right)} I_{W / A-1}(2 \sqrt{F / A})}{I_{W / A-1}(2 \sqrt{2 F / A})} .
$$


Therefore,

$$
P(n)=\frac{2^{\frac{1}{2}\left(\frac{W}{A}-1\right)}}{n !}\left(\sqrt{\frac{F}{A}}\right)^{n} \frac{I_{W / A+n-1}(2 \sqrt{F / A})}{I_{W / A-1}(2 \sqrt{2 F / A})},
$$

in agreement with Ref. [44], where the solution was obtained using a generating function. To examine the effect of the cutoff at $s \leq S$, we compared the distributions $P(n)$ obtained from Eqs. (32) for different values of $s$, as well as the distribution expressed in terms of the Bessel functions for which $s \rightarrow \infty$. Under the conditions of low coverage studied here we observe a very fast convergence of Eq. (32) to Eq. (36) as $s$ increases. We have also performed direct numerical integration of the master equation and found that the solution given by Eq. (32) is stable and the convergence of the integration process is fast.

Using similar summations, we can now find exact expressions for the first and second moments of the distribution $P(n)$. The average number of $\mathrm{H}$ atoms on the grain is given by

$$
\langle n\rangle=\sqrt{\frac{F}{2 A}} \frac{I_{W / A}(2 \sqrt{2 F / A})}{I_{W / A-1}(2 \sqrt{2 F / A})} .
$$

The rate of formation of $\mathrm{H}_{2}$ molecules is given by $R=A\langle n(n-1)\rangle$, where

$$
\langle n(n-1)\rangle=\frac{F}{2 A} \frac{I_{W / A+1}(2 \sqrt{2 F / A})}{I_{W / A-1}(2 \sqrt{2 F / A})},
$$

and the recombination efficiency is

$$
\eta=\frac{I_{W / A+1}(2 \sqrt{2 F / A})}{I_{W / A-1}(2 \sqrt{2 F / A})}
$$

The second moment $\langle n 2\rangle$ can be obtained as the sum of the right hand sides of Eqs. (37) and (38). The fluctuations in the number of $\mathrm{H}$ atoms on different grains can be quantified by the standard deviation of the distribution $P(n)$, given by

$$
\sigma=\sqrt{\left\langle n^{2}\right\rangle-\langle n\rangle^{2}}
$$

The limits of first and second order kinetics for large grains can now be reproduced from Eq. (39). The extreme limit of first order kinetics is characterized by negligible desorption, namely $W / A \ll 1$ and for large enough grains this limit also satisfies $F / W \gg 1$. In this limit the indices of the Bessel functions in Eq. (39) $W / A \pm 1 \rightarrow \pm 1$, respectively, and due 
to the symmetry $I_{-n}(z)=I_{n}(z)$ of the Bessel functions 49 (Eq. 9.6.6 in page 375) $\eta \rightarrow 1$, in agreement with the rate equations. The extreme limit of second order kinetics on large grains is characterized by $F / W \ll W / A$. Assuming that the geometric mean of $F / W$ and $W / A$ satisfies $\sqrt{F / A} \ll 1$ we obtain

$$
\eta=\frac{2 F}{W} \frac{1}{\left(\frac{W}{A}-1\right)} .
$$

In the case of very large grains, namely $W / A \gg 1$ we thus obtain $\eta=2 A F / W 2=2 a f / W 2$ which is in agreement with the rate equation results.

Using Eqs. (23) and (22) to express the time derivative of $\langle n\rangle$ we obtain

$$
\frac{d\langle n\rangle}{d t}=f-W\langle n\rangle-2 A\langle n(n-1)\rangle .
$$

This equation resembles the rate equation (20) except for the recombination term in which $\langle n\rangle 2$ was replaced by $\langle n 2\rangle-\langle n\rangle$. In the limit of small grains, where $\langle n\rangle$ is small while the fluctuations represented by $\sigma$ become dominant, the rate equation becomes unsuitable and over-estimates the rate of $\mathrm{H}_{2}$ production.

\section{Analysis and Results}

Consider the recombination process on a small grain with $S$ adsorption sites under steady state conditions. The boundary between the first order and the second order regimes can be expressed in terms of $F=f \cdot S$ and $A=a / S$, taking the form $F / W=W / A$ (neglecting the $\mathrm{LH}$ rejection). In the first order regime $F / W \gg W / A$, while in the second order regime $F / W \ll W / A$. The domains of first and second order kinetics are shown in Fig. 5, separated by the diagonal line $F / W=W / A$.

The finite size of the grain introduces a third length scale to the problem. We will now examine how small the grain should be in order for the recombination efficiency to deviate significantly from the rate equation results. To this end we calculate $\eta$ as a function of

the grain size $S$, using the exact solution of the master equation, presented above. The efficiency $\eta$ vs. $S$ in the case of first order kinetics is shown in Fig. 6(a). The recombination efficiency obtained from the master equation (solid line) coincides with the rate equation result (dashed line) for large grains but declines below some grain size. In the first order 
case, such deviations typically occur only for extremely small grains of a few thousands adsorption sites. The average number of atoms on the grain vs. $\mathrm{S}$ is shown in Fig. 6(b). The $S$ axis in Figs. 6 corresponds to the arrow drawn in the first order domain of Fig. 5 . Identifying the corresponding symbols, we observe that the significant decline in $\eta$ starts when $F / W<1$, namely when $S<W / f$. The fluctuations in the number of $\mathrm{H}$ atoms on a grain can be quantified by the standard deviation $\sigma$ of the distribution $P(n)$, given by Eq. (40). The standard deviation $\sigma$, divided by $\langle n\rangle$, is shown in Fig. 6(c) as a function of the grain size $S$. Clearly, as the grain size decreases, the fluctuations become more pronounced.

Using the notation introduced above, in the domain of first order kinetics the rate equation results start to deviate from the correct value of $\eta$ for grain sizes that satisfy

$$
S<s_{\text {vacant }}<s_{\text {visit }}
$$

Under these conditions there is typically no atom or only a single $\mathrm{H}$ atom on the grain, which is unlikely to find a second atom to recombine with. It thus desorbs before it has a chance to recombine.

The efficiency $\eta$ in the case of second order kinetics is shown in Fig. 7(a). Again, the master equation efficiency (solid line) coincides with the rate equation result (dashed line) for large grains but declines below some grain size, typically of a few tens of thousands of adsorption sites. The average number of atoms on the grain vs. $\mathrm{S}$ is shown in Fig. 7(b). The deviations between the rate equations and the master equation results are accompanied by large fluctuations in the number of atoms on a grain, as can be seen in Fig. 7(c). The $S$ axis in Figs. 7 corresponds to the arrow drawn in the second order domain of Fig. 5 . Identifying the corresponding symbols, we observe that the significant decline in $\eta$ starts when $W / A<1$, namely $S<a / W$. Thus, in the domain of second order kinetics, deviations between the rate equations and the master equation occur for a range of grain sizes given by

$$
S<s_{\text {visit }}<s_{\text {vacant }}
$$

In this case, the grain surface area is smaller than the area that an atom can scan before it desorbs. As a result the atom tends to perform several sweeps of the grain surface visiting again the same vacant sites it has already visited. The probability to find a second atom 
in these sites is much lower than predicted by the rate equation that does not include such return visits. The recombination efficiency is thus sharply reduced as the grain size further decreases.

In conclusion, we observe that in both the first and second order kinetics the recombination efficiency on a grain (given by the master equation) starts to deviate from the rate equation result when the grain size (represented by $S$ ) becomes the smallest length scale in the problem. In the first order case this happens when $S<s_{\text {vacant }}$ while in the second order case it happens when $S<s_{\text {visit }}$.

The distribution $P(n)$ at three points along the $S$ axis, in the second order domain, are shown in Fig. 8. For a relatively large grain, $P(n)$ exhibits a well defined and nearly symmetric peak. For a very small grain it becomes a a monotonically decreasing function, dominated by $n=0,1$ and 2 .

\section{DISCUSSION}

The analysis above can be related to the modified rate equations studied in Refs. [36, 37, 38]. The modification is needed when the grain size becomes smaller than the two length scales involved in the recombination process. In the case of first order kinetics this occurs when $S<W / f<a / W$, while in second order desorption it occurs when $S<a / W<W / f$.

Consider the case of first order kinetics. When the grain becomes smaller than $W / f$ the typical number of atoms on the grain is smaller than one (even if we consider the depletion of the $\mathrm{H}$ atoms on the surface due to desorption alone and neglect recombination). Therefore, the rate in which atoms find each other on the surface is not determined anymore by the hopping rate $a$, or the corresponding length scale $a / W$, but by the grain size $S$. Therefore, the recombination term in Eq. (20) should be modified to reflect the change $a / W \rightarrow S$, or equivalently $A / W \rightarrow 1$. This is achieved by replacing $A$ by $W$.

In the case of second order kinetics, when the grain size becomes smaller than $a / W$ the atom is typically able to perform more than a full sweep of the entire grain before it desorbs. However, when an atom visits the same sites for the second time, the probability of finding another $\mathrm{H}$ atom there is greatly reduced. Therefore, the recombination rate is determined by $\mathrm{S}$ rather than by $a / W$, requiring the modification $a / W \rightarrow S$, or the replacement of $A$ by $W$ in Eq. (20), exactly as in the case of first order kinetics. The modified rate equations 
thus takes the form

$$
\frac{d\langle n\rangle}{d t}=F-W\langle n\rangle-2 g\left(\frac{A}{W}, \frac{W}{F}\right)\langle n\rangle^{2}
$$

where

$$
g\left(\frac{A}{W}, \frac{W}{F}\right)=\left\{\begin{array}{l}
A: \frac{A}{W}<1 \text { or } \frac{W}{F}<1 \\
W: \frac{A}{W}>1 \text { and } \frac{W}{F}>1
\end{array}\right.
$$

The modified rate equations takes into account correctly the length scales involved in the recombination process on small grains. It provides results for $\langle n\rangle$ that are in significantly better agreement with the master equation, compared to the unmodified rate equations. It also improves the results for the recombination efficiency. However, the modified rate equations still involve only the average number of atoms on a grain and do not take into account the discreteness of the $\mathrm{H}$ atoms and the fluctuations, quantified by the second moment in Eq. (42). Since the fluctuations dominate the recombination process on small grains, the results of the modified rate equations for $\eta$ are not expected to coincide with those of the master equation, but only to approximate them better than the ordinary rate equations. We observe that the deviations between the rate equation results and the correct results obtained from the master equation are significant mostly in the second order domain [Fig. 7]. In the domain of first order kinetics such deviations occur only for extremely small grains, that may be physically irrelevant [Fig. 6].

While the results of the calculations above were presented using dimensionless parameters, the actual calculations in Figs. 6 and 7 were done for physically relevant parameters. In both of them we used the parameters of the amorphous carbon sample, measured experimentally in Refs. [15, 33]. On this sample the activation energies for $\mathrm{H}$ diffusion and desorption were found to be $E_{0}=44.0 \mathrm{meV}$ and $E_{1}=56.7 \mathrm{meV}$, respectively. The density of adsorption sites on the amorphous carbon surface was found to be $s_{d e n s} \cong 5 \times 10^{13}\left(\right.$ sites $\left.\mathrm{cm}^{-2}\right)$. The results for first order kinetics (Fig. 6) were obtained for $T=17 \mathrm{~K}$ and $f=5 \times 10^{-8} \mathrm{ML} \mathrm{s}^{-1}$. The results for second order kinetics (Fig. (7) were obtained for $T=18 \mathrm{~K}$ and $f=3.4 \times 10^{-9}$ $\mathrm{ML} \mathrm{s}^{-1}$. The connection with the density and temperature of the hydrogen in the gas phase is made through $f=\rho_{\text {gas }} v_{\text {gas }} / 4 s_{\text {dens }}$ where $\rho_{\text {gas }}\left(\right.$ atoms $\left.\mathrm{cm}^{-3}\right)$ is the density of $\mathrm{H}$ atoms in the gas phase, $v_{\text {gas }}$ is the typical velocity of these atoms and the factor or 4 in the denominator is the ratio between the surface area and the cross section for a spherical grain [43]. The 
number of adsorption sites on a spherical grain of diameter $d$ is given by

$$
S=4 \pi\left(\frac{d}{2}\right)^{2} \cdot s_{\mathrm{dens}},
$$

and the flux $F=\pi(d / 2)^{2} \rho_{\text {gas }} v_{\text {gas }}$. Observations indicate that the population of carbonaceous and silicate grains in interstellar clouds exhibits a broad distribution of grain sizes, roughly in the range $10^{-6} \mathrm{~cm}<d<10^{-4} \mathrm{~cm} \mathrm{[50,} \mathrm{51,} \mathrm{52,} \mathrm{53]}$

\section{SUMMARY}

We have studied the process of hydrogen recombination on small grains in the interstellar medium using the master equation approach. An exact solution of the master equation under steady state conditions was presented. This solution provides the probability distribution $P(n)$ of having $n$ atoms on the grain as a function of the grain size, flux, temperature and the parameters of the surface. From this distribution one can obtain an exact expression for the hydrogen recombination rate on the grain surface. The results were compared to those obtained from the rate equations that describe the recombination process on macroscopic surfaces. In the case of a macroscopically large surface, two length scales are identified. One length scale is related to the average number of vacant sites around each adsorbed atom, given by $s_{\text {vacant }}$. The other length scale is given by the average number of sites, $s_{\text {visit }}$, that an adsorbed atom visits before it desobs (neglecting recombination). The relation between these two (length) scales determines the properties of the recombination process, dividing the parameter space into two domains. The domain $s_{\text {visit }}>s_{\text {vacant }}$ is characterized by first order kinetics with high recombination efficiency, while the domain $s_{\text {visit }}<s_{\text {vacant }}$ exhibits second order kinetics with low recombination efficiency. In both domains the finite size of the grain enters as a significant factor (requiring the use of the master equation rather than the rate equations) when it becomes the smallest (length) scale in the system. In the domain of first order kinetics the grain size becomes the smallest (length) scale when $S<s_{\text {vacant }}$ while in the second order it occurs when $S<s_{\text {visit }}$.

Acknowledgements: We thank I. Furman, E. Herbst, H. Mehl, V. Pirronello, A. Schiller and G. Vidali for helpful discussions. This work was supported by the Adler Foundation for 
Space Research of the Israel Science Foundation.

[1] R.J. Gould and E.E. Salpeter, Astrophys. J. 138, 393 (1963).

[2] D. Hollenbach and E.E. Salpeter, J. Chem. Phys. 53, 79 (1970).

[3] D. Hollenbach and E.E. Salpeter, Astrophys. J. 163, 155 (1971).

[4] D. Hollenbach, M.W. Werner and E.E. Salpeter, Astrophys. J. 163, 165 (1971).

[5] D.A. Williams, Astrophys. J. 151, 935 (1968).

[6] R. Smoluchowski, Astrophys. Space Sci. 75, 353 (1981).

[7] R. Smoluchowski, J. Chem. Phys. 87, 4229 (1983).

[8] S. Aronowitz and S.B Chang, Astrophys. J. 293, 243 (1985).

[9] W.W. Duley and D.A. Williams, D.A., Mon. Not. R. Astron. Soc. 223, 177 (1986).

[10] V. Pirronello and D. Averna, Astron. Astrophys. 201, 196 (1988).

[11] S.A. Sandford and L.J. Allamandolla, Astrophys. J. 409, L65 (1993).

[12] J. Takahashi, K. Masuda and M. Nagaoka, Mon. Not. R. Astron. Soc. 306, 22 (1999).

[13] V. Pirronello, C. Liu, L. Shen and G. Vidali, Astrophys. J. 475, L69 (1997).

[14] V. Pirronello, O. Biham, C. Liu, L. Shen and G. Vidali, Astrophys. J. 483, L131 (1997).

[15] V. Pirronello, C. Liu, J.E. Roser and G. Vidali, Astron. Astrophys. 344, 681 (1999).

[16] G. Manicò, G. Ragunì, V. Pirronello, J.E. Roser and G. Vidali, Astrophys. J. 548, L253 (2001).

[17] R.T. Brackmann and W.L. Fite, J. Chem. Phys. 34, 1572 (1961).

[18] A. Schutte, D. Bassi, F. Tommasini, F. Turelli, G. Scoles and L.J.F. Herman, J. Chem. Phys. 64, 4135 (1976).

[19] V. Buch and Q. Zhang, Astrophys. J. 379, 647 (1991).

[20] V. Buch and R. Czerminski, J. Chem. Phys. 95, 6026 (1991).

[21] J.B. Pickles and D.A. Williams, Astrophys.\& Space Science 52, 433 (1977).

[22] L.B. d'Hendecourt, L.J. Allamandola and J.M. Greenberg, Astron. Astrophys. 152, 130 (1985).

[23] P.D. Brown, Mon. Not. R. Astron. Soc. 243, 65 (1990).

[24] P.D. Brown and S.B. Charnley, Mon. Not. R. Astron. Soc. 244, 432 (1990).

[25] T.I. Hasegawa and E. Herbst and C.M. Leung, ApJ Supplement 82, 167 (1992). 
[26] T.I. Hasegawa and E. Herbst, Mon. Not. R. Astron. Soc. 261, 83 (1993).

[27] T.I. Hasegawa and E. Herbst, Mon. Not. R. Astron. Soc. 263, 589 (1993).

[28] P. Caselli, T.I. Hasegawa and E. Herbst, Astrophys. J. 408, 548 (1993).

[29] P. Caselli, T.I. Hasegawa and E. Herbst, Astrophys. J. 421, 206 (1994).

[30] D.P. Ruffle and E. Herbst, Mon. Not. R. Astron. Soc. 319, 837 (2000).

[31] D.P. Ruffle and E. Herbst, Mon. Not. R. Astron. Soc. 322, 770 (2001).

[32] D.P. Ruffle and E. Herbst, Mon. Not. R. Astron. Soc. 324, 1054 (2001).

[33] N. Katz, I. Furman, O. Biham, V. Pirronello and G. Vidali, Astrophys. J. 522, 305 (1999).

[34] A.G.G.M. Tielens, 1995, unpublished.

[35] S.B. Charnley, A.G.G.M. Tielens and S.D. Rodgers, Astrophys. J. 482, L203 (1997).

[36] P. Caselli, T.I. Hasegawa and E. Herbst, Astrophys. J. 495, 309 (1998).

[37] O.M. Shalabiea, P. Caselli and E. Herbst, Astrophys. J. 502, 652 (1998).

[38] T. Stantcheva, P. Caselli and E. Herbst, Astron. Astrophys. 375, 673 (2001).

[39] S.B. Charnley, Astrophys. J. 509, L121 (1998).

[40] S.B. Charnley, Astrophys. J. 562, L99 (2001).

[41] M.E.J. Newman and G.T. Barkema, Monte Carlo methods in statistical physics (Clarendon Press, Oxford, 1999).

[42] N.G. van Kampen, Stochastic Processes in Physics and Chemistry (North-Holland, Amsterdam, 1981).

[43] O. Biham, I. Furman, V. Pirronello and G. Vidali, Astrophys. J. 553, 595 (2001).

[44] N.J.B. Green, T. Toniazzo, M.J. Pilling, D.P. Ruffle, N. Bell and T.W. Hartquist, Astron. Astrophys. 375, 1111 (2001).

[45] T. Stantcheva, V.I. Shematovich and E. Herbst, On the master equation approach to diffusive grain-surface chemistry: the H,O,CO system, preprint (2002).

[46] A. Zangwill, Physics at Surfaces (Cambridge University Press, Cambridge, 1988).

[47] E.W. Montroll and G.H. Weiss, J. Math. Phys. 6, 167 (1965).

[48] O. Biham, I. Furman, N. Katz, V. Pirronello and G. Vidali, Mon. Not. R. Astron. Soc. 296, 869 (1998).

[49] M. Abramowitz and I.A. Stegun, Handbook of Mathematical Functions (Dover, New York, 1965).

[50] J.S. Mathis, W. Rumpl and K.H. Nordsieck, Astrophys. J. 217, 425 (1977). 
[51] J.S. Mathis, Astrophys. J. 472, 643 (1996).

[52] J.E. O'Donnell and J.S. Mathis, Astrophys. J. 479, 806 (1997).

[53] J.C. Weingartner and B.T. Draine, Astrophys. J. 548, 296 (2001). 
FIG. 1: (a) The phase diagram of hydrogen recombination on macroscopic surfaces in the $(W / a, f / W)$ plane, as described by Eq. (11). The parameter space is divided into two domains: the first order domain, above the diagonal and the second order domain below it;

FIG. 2: (a) The coverage $\rho$ given by Eq. (6) as a function of the angle $\theta$, given by Eq. (10) (see Fig. 1), without LH rejection (solid line) and with LH rejection (dashed line). The results shown are for points along the curve given by Eq. (11). (b) The recombination efficiency $\eta$ [Eq. (7)] vs. $\theta$, without LH rejection (solid line) and with LH rejection (dashed line), along the same curve. Note that without LH rejection $\eta$ depends only on $\theta$, namely, it is independent of the specific curve.

FIG. 4: The phase diagram of the hydrogen recombination process on macroscopic surfaces in the $(T, \ln f)$ plane under the LH kinetics. The strip of high efficiency is now bounded from both sides, namely for any given flux there is a range of temperatures that exhibits high recombination efficiency.

FIG. 5: The phase diagram of the hydrogen recombination process on grains without LH rejection, in terms of the dimensionless parameters $W / A$ and $F / W$. The diagonal line separates between the domain of first order (on the left) and second order recombination (on the right), respectively. The (unit) square near the origin is the domain in which the hydrogen recombination efficiency on grains (obtained from the master equation) deviates significantly from the rate equation results. In the case of first order processes, the condition for such deviation is $F / W<1$, while in second order processes the condition is $W / A<1$. Also included are two axes, in the first and the second order domains, that under given physical conditions (fixed values of $f$ and $T$ ) represent the variation in the grain size. The behavior of $\eta,\langle n\rangle$ and $\sigma /\langle n\rangle$ along these axes is shown in Figs. 6 and 0 for the first and second order cases, respectively.

FIG. 3: The phase diagram of hydrogen recombination on macroscopic surfaces under the LH kinetics. The high efficiency domain is bounded to the gray area, on the left side of the curve given by Eq. (17). 
FIG. 6: The hydrogen recombination efficiency $\eta$ (a) and the average number of atoms on the grain $\langle n\rangle$ (b) for first order kinetics as a function of the number of adsorption sites, $S$, on the grain. The solid line (with the symbols on it) shows the results obtained from the master equation, and the dashed line shows the rate equation results. The symbols correspond to those in Fig. 5, and indicate that the deviations between the master equation and the rate equations become significant below $F / W=1$. (c) The standard deviation $\sigma$ of the distribution $P(n)$ (normalized by $\langle n\rangle$ ). It increases sharply as the grain size is reduced entering the range in which $F / W<1$, indicating that in this range fluctuations play an important role.

FIG. 7: The hydrogen recombination efficiency $\eta$ (a) and the average number of atoms on the grain $\langle n\rangle$ (b) for second order kinetics as a function of the number of adsorption sites, $S$, on the grain. The solid line (with the symbols on it) show the results obtained from the master equation, and the dashed line show the rate equation results. The symbols correspond to those in Fig. 周, and indicate that the deviation between the master equation and the rate equations become significant below $W / A=1$. (c) The standard deviation $\sigma$ of the distribution $P(n)$ (normalized by $\langle n\rangle$ ). It increases sharply as the grain size is reduced entering the range in which $W / A<1$, indicating that in this range fluctuations play an important role.

FIG. 8: The distribution $P(n)$ at three points along the arrow in the second order domain of Fig. 5, namely for a small grain with $S=2513$ (solid line), and medium size grain with $S=56705$ (dashed line) and for a large grain with $S=141372$. 


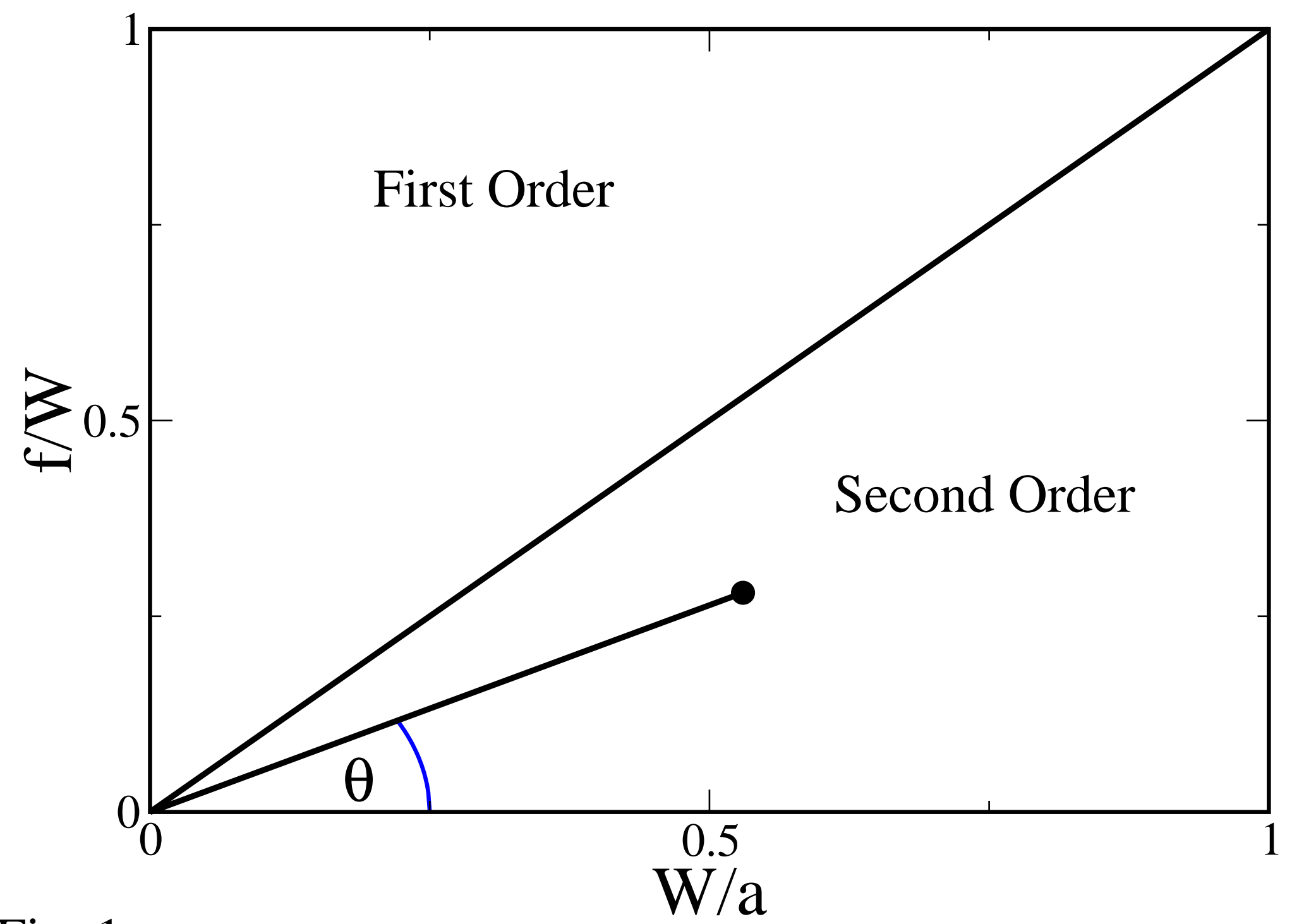

Fig. 1 


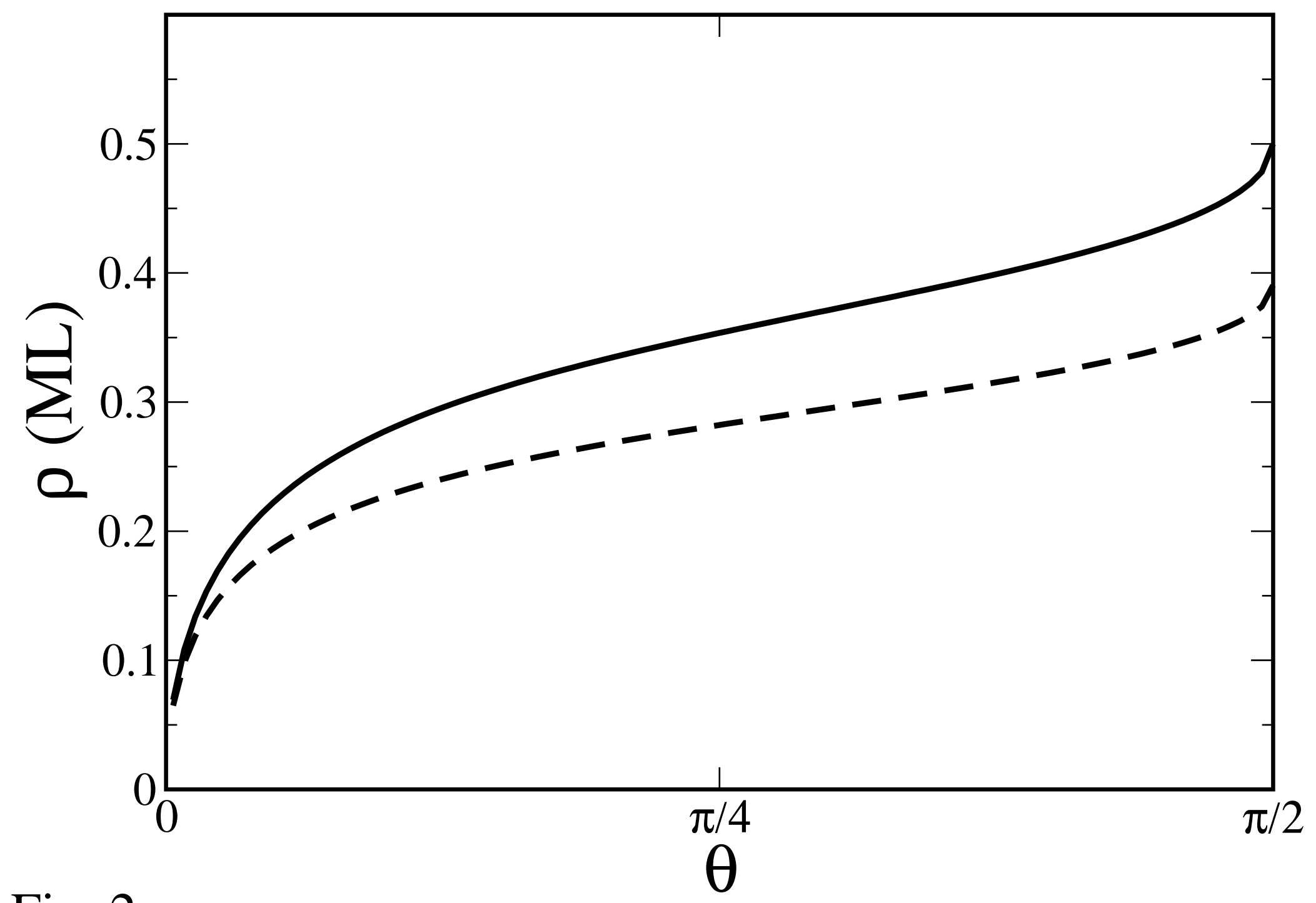

Fig. 2a 


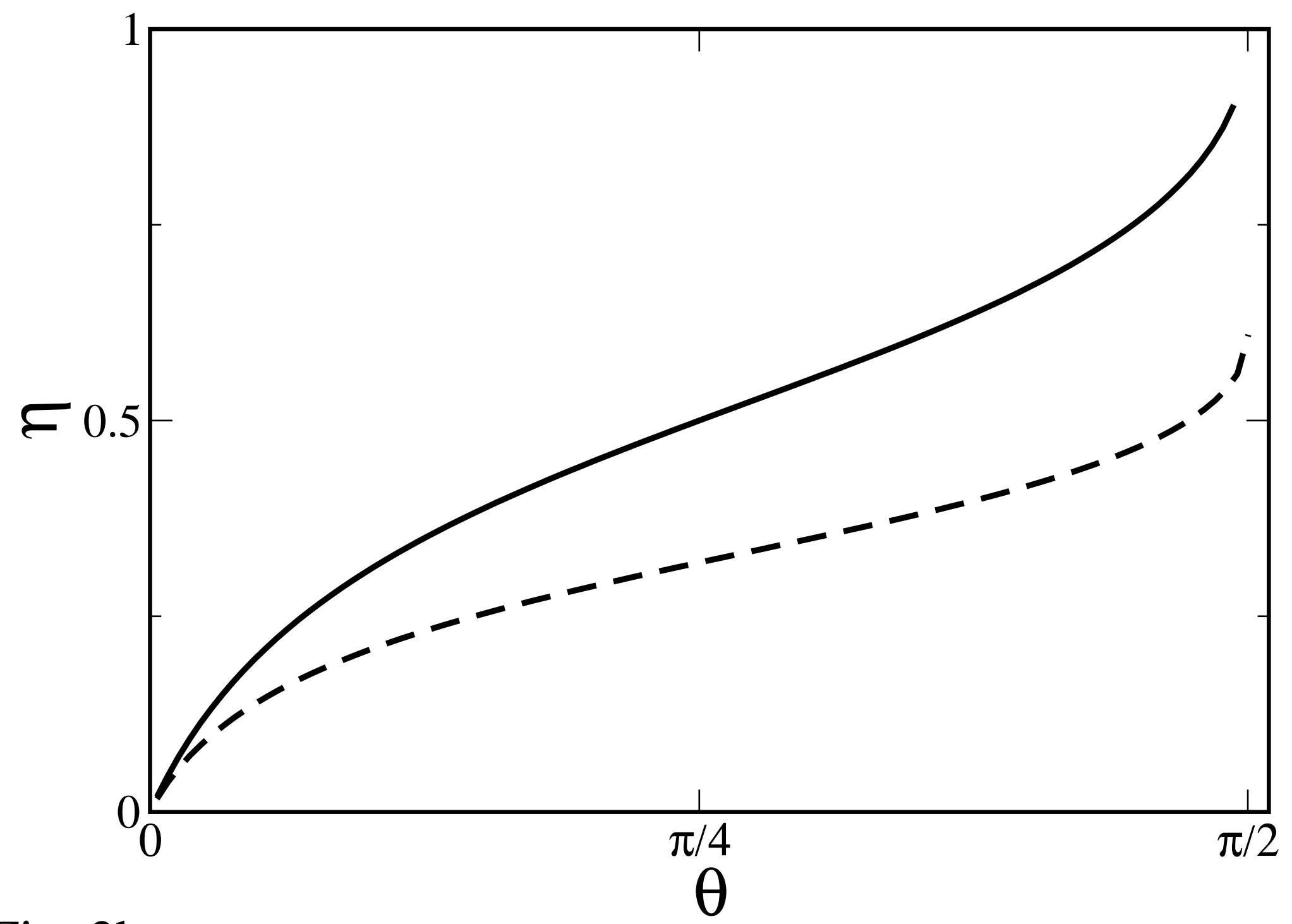

Fig. 2b 


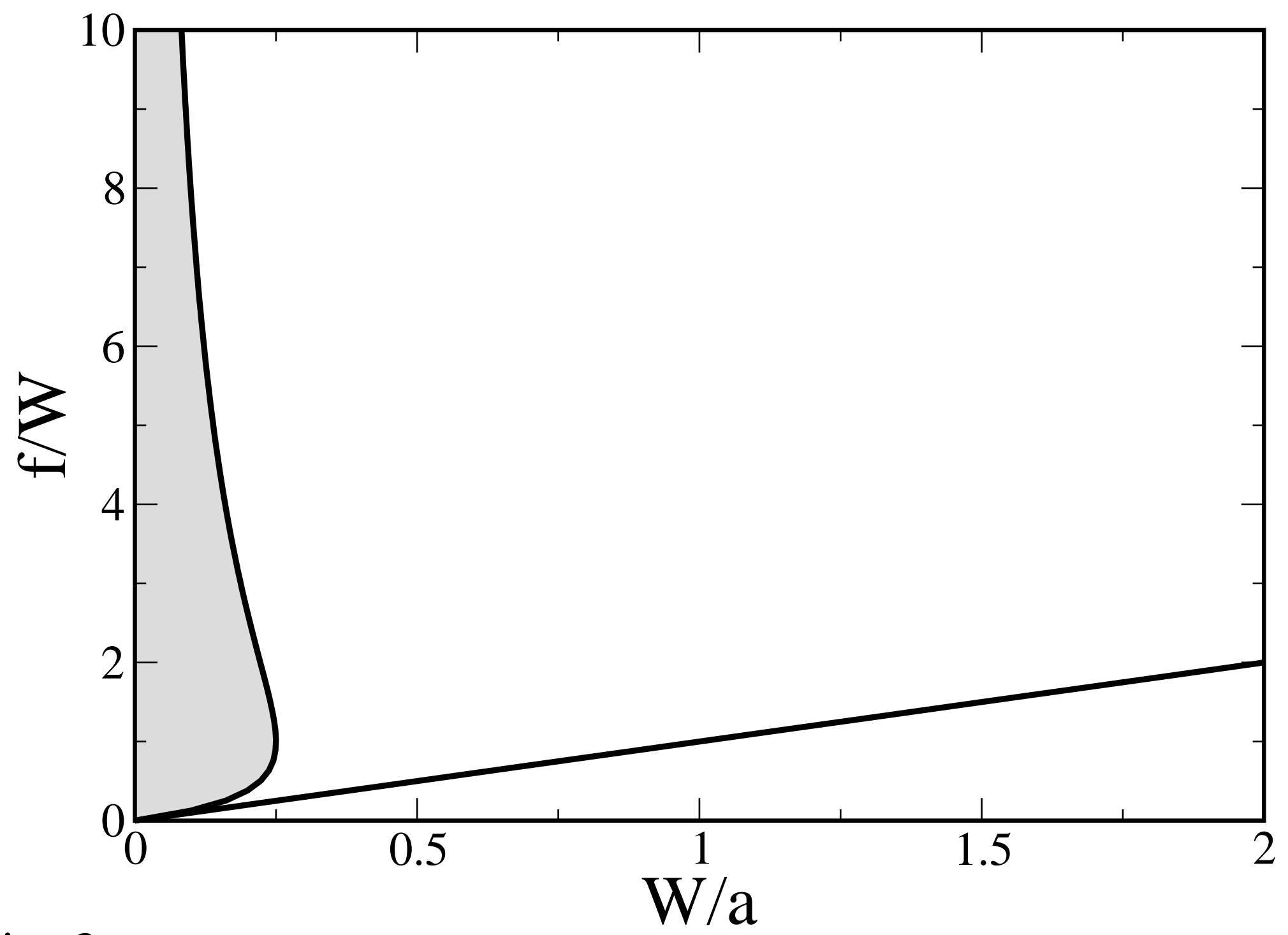

Fig. 3 
Fig. 5

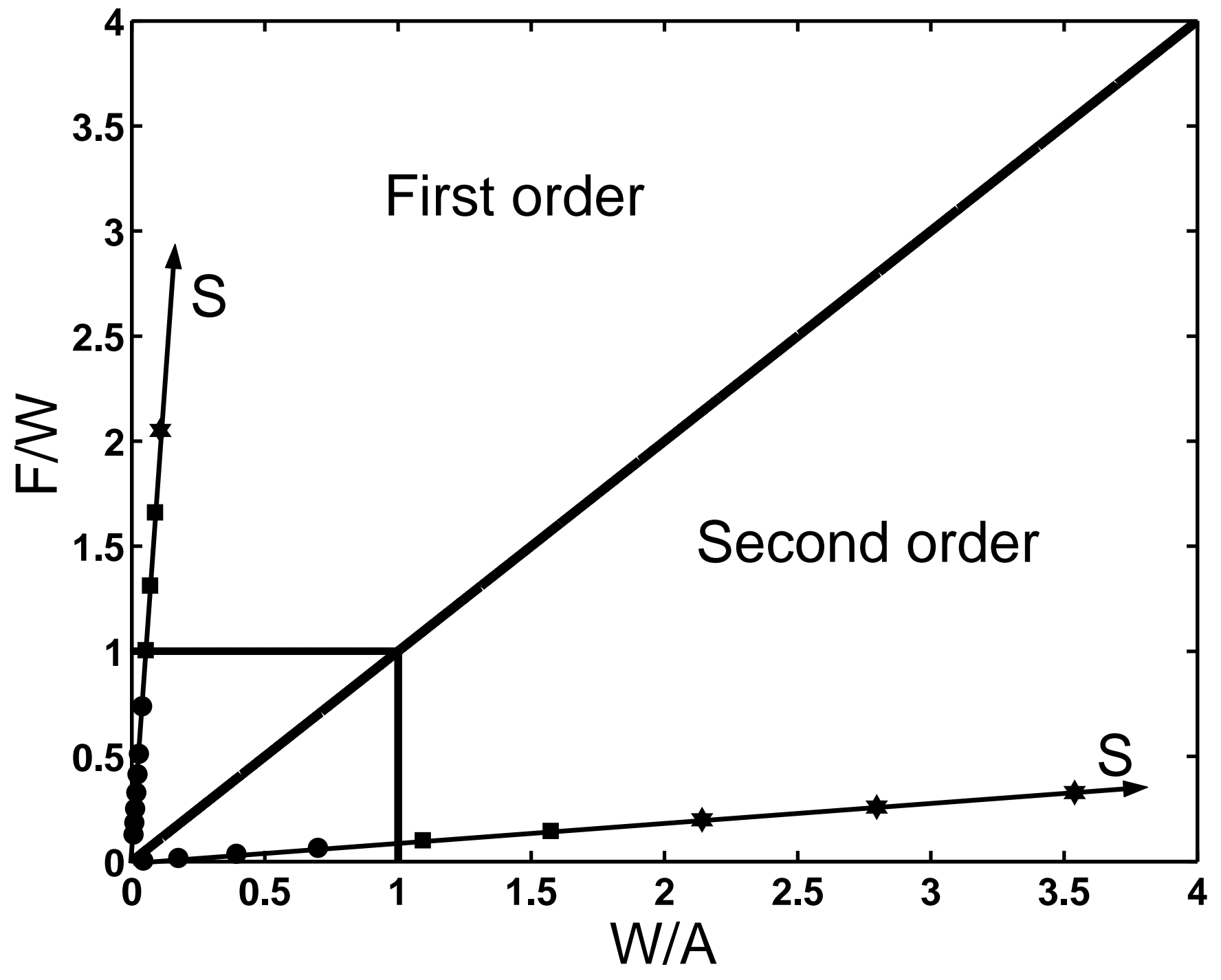




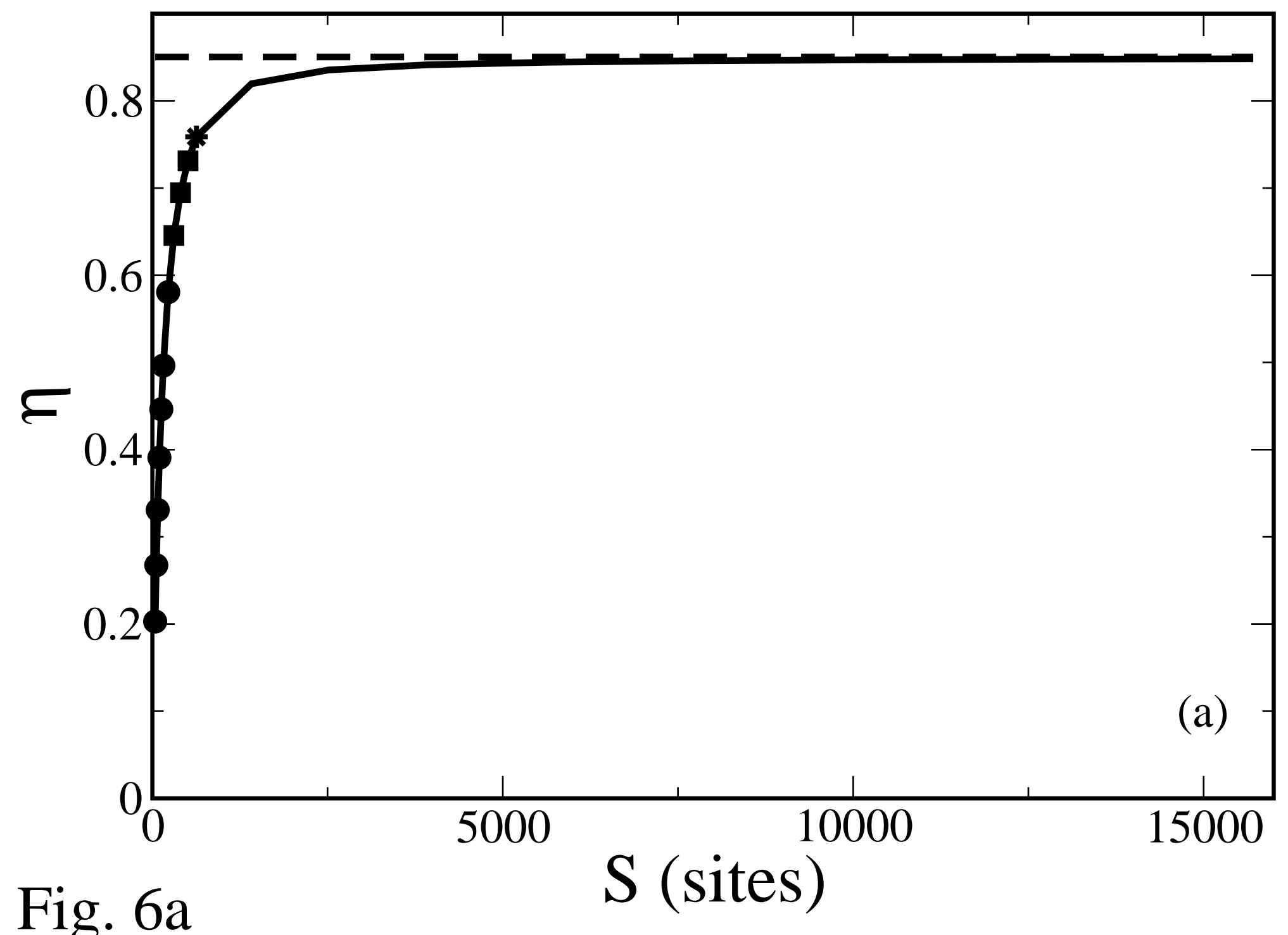

Fig. 6a 


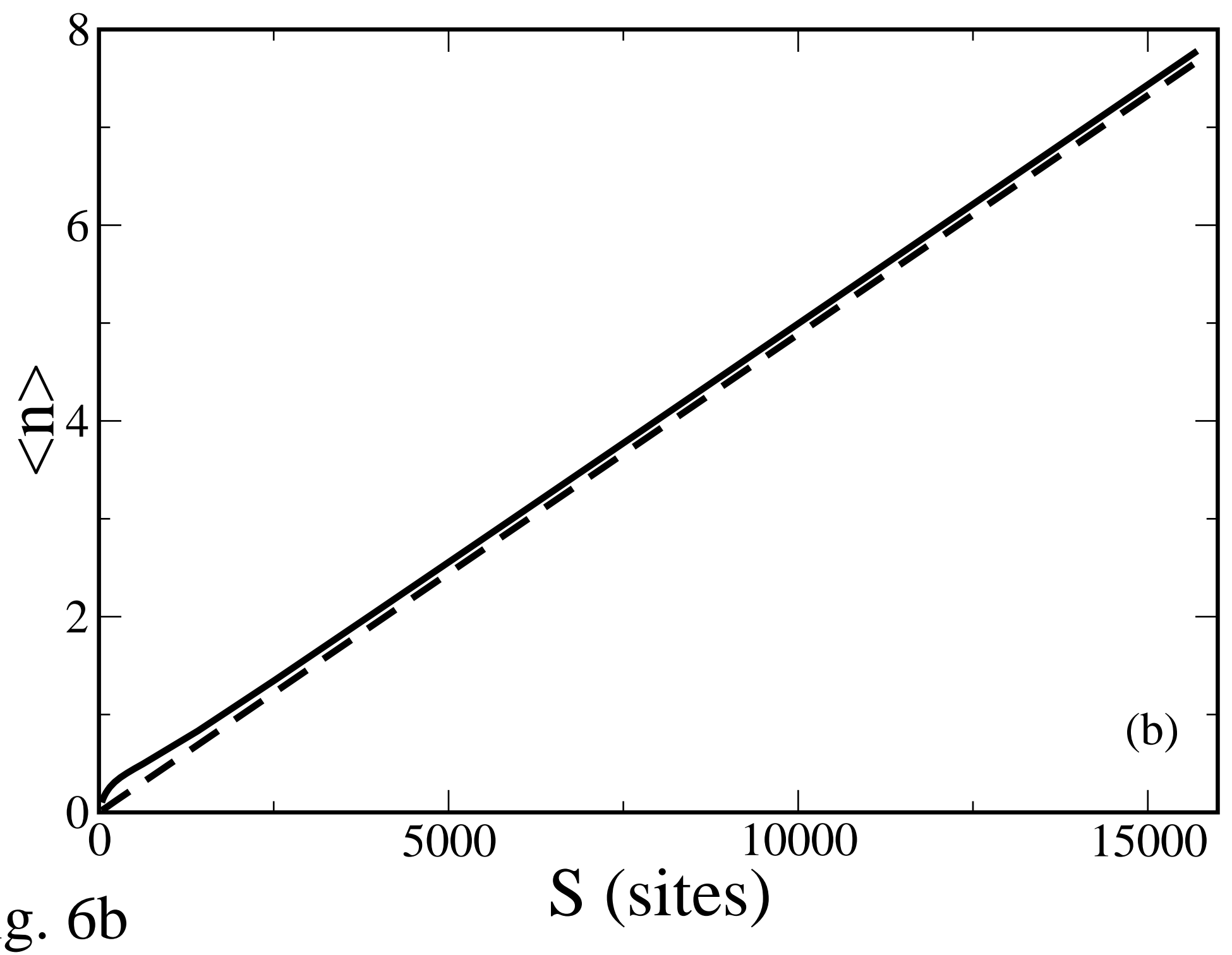




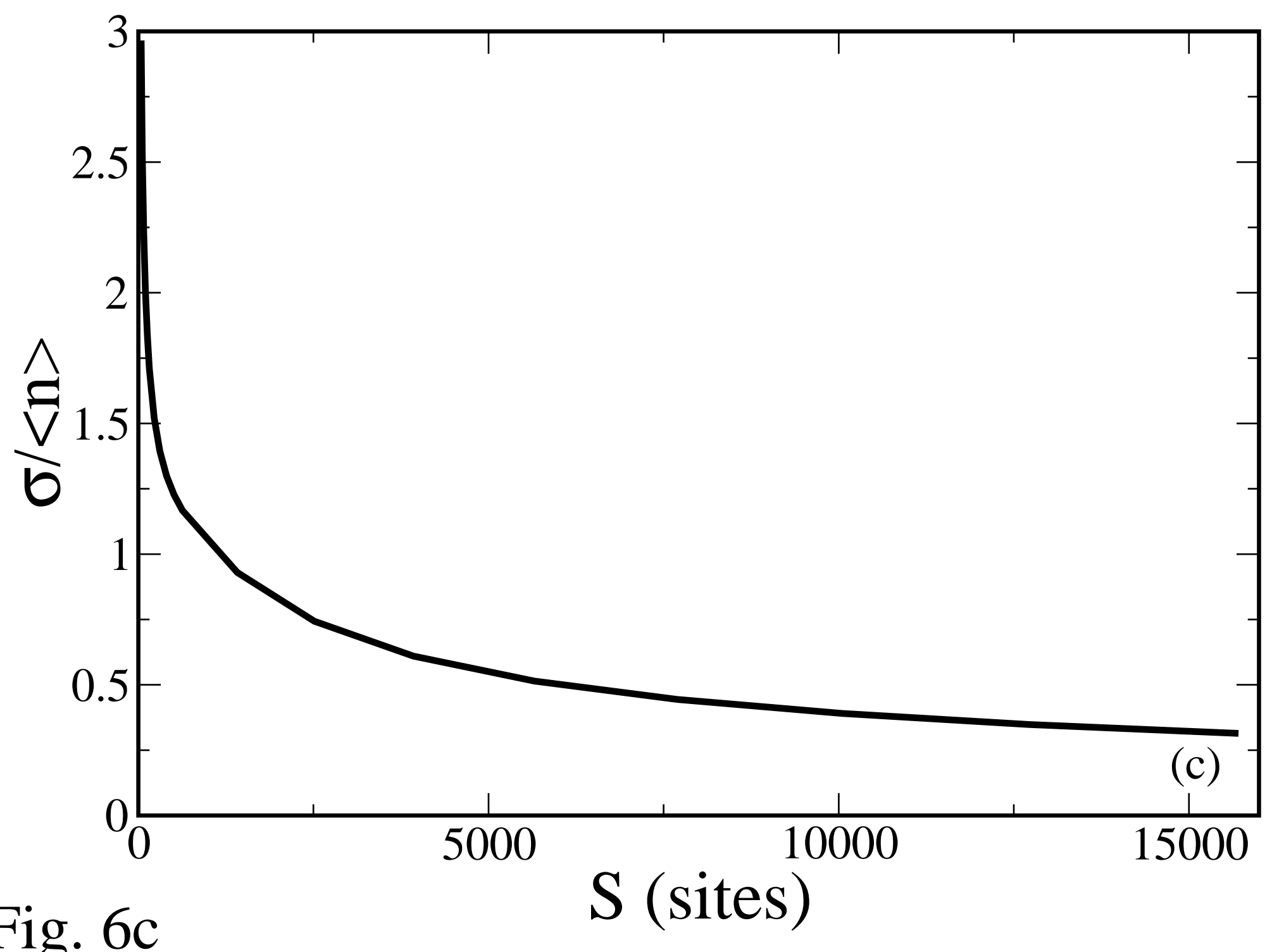

Fig. 6c 


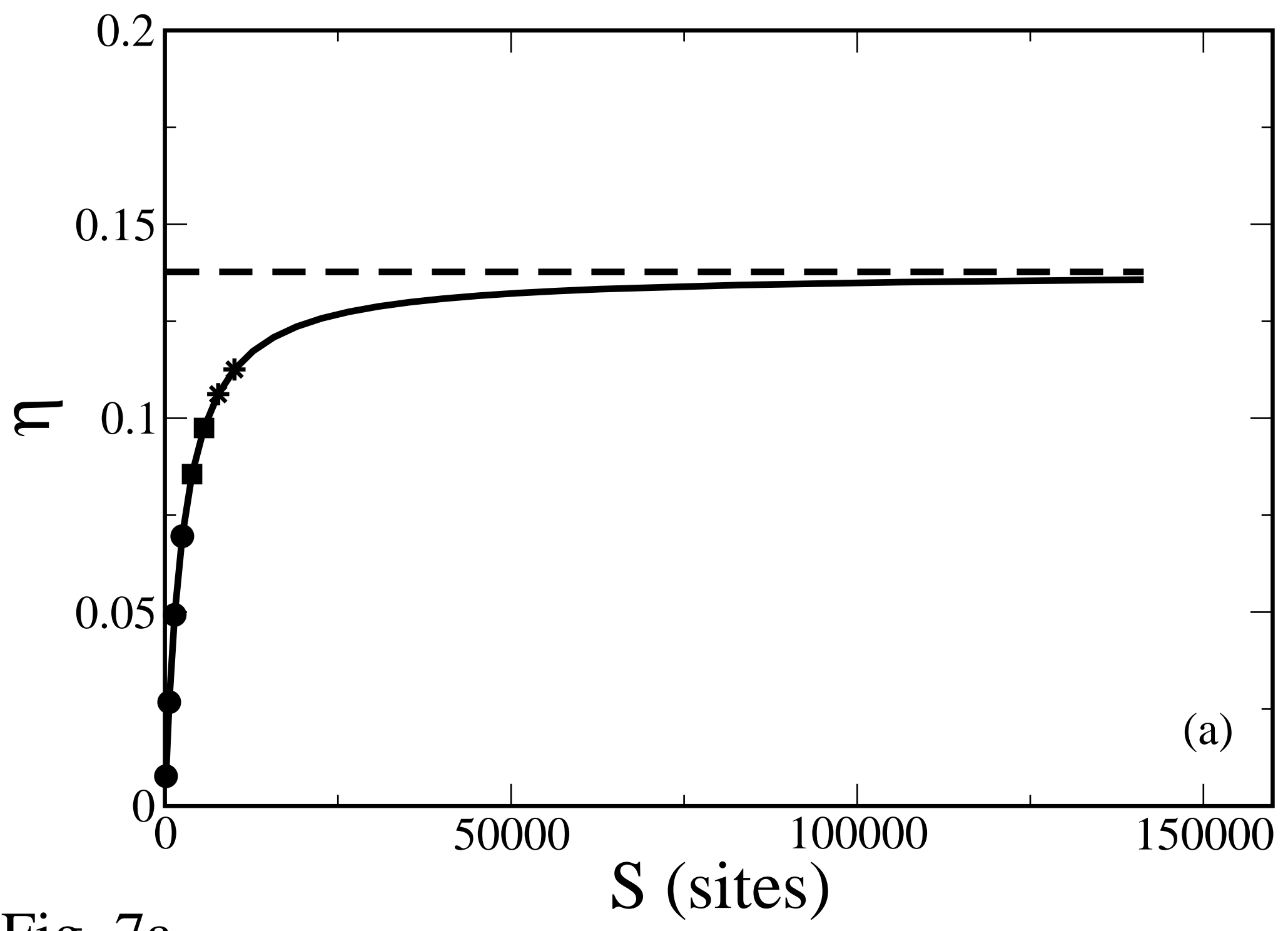

Fig. 7a 


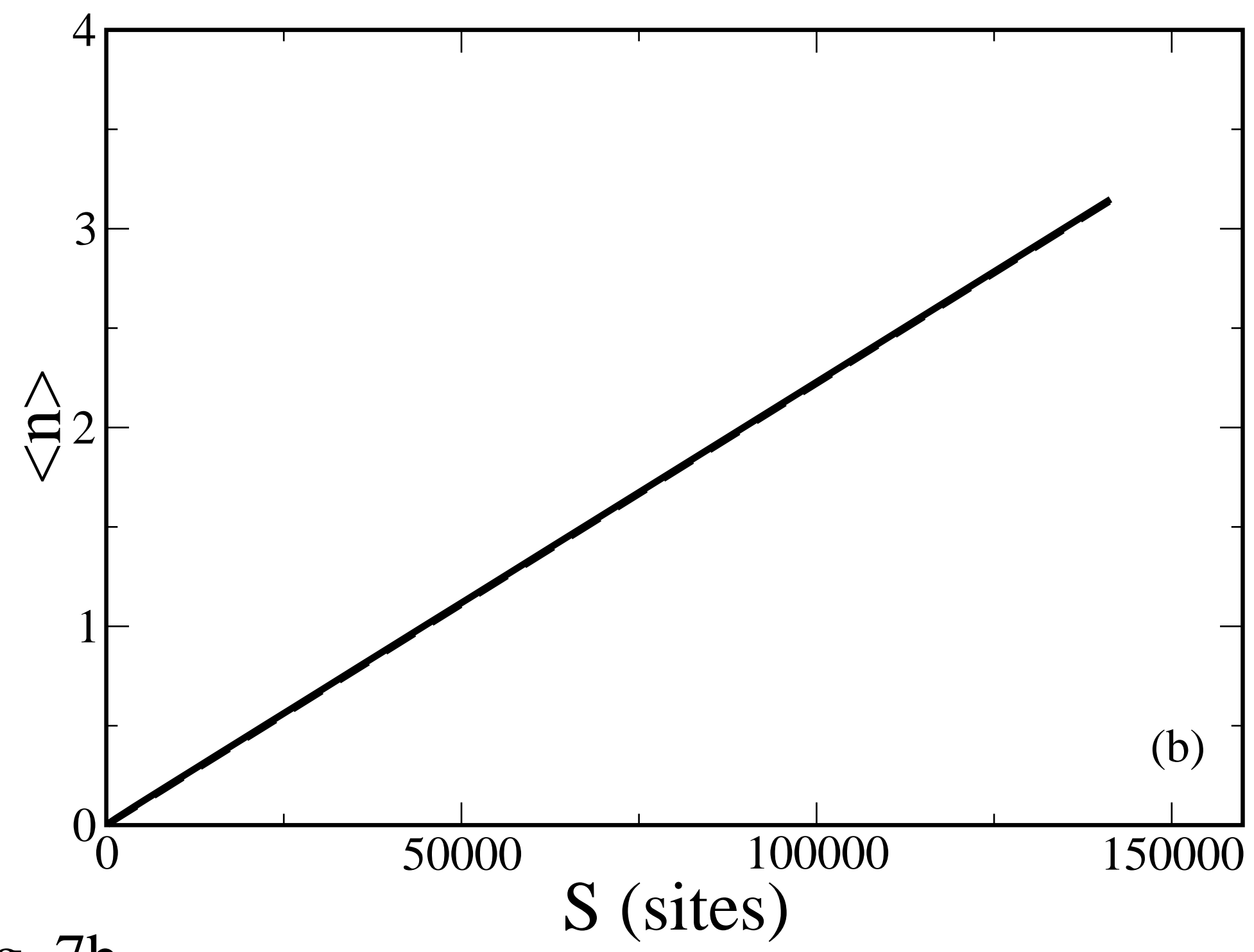

Fig. 7b 


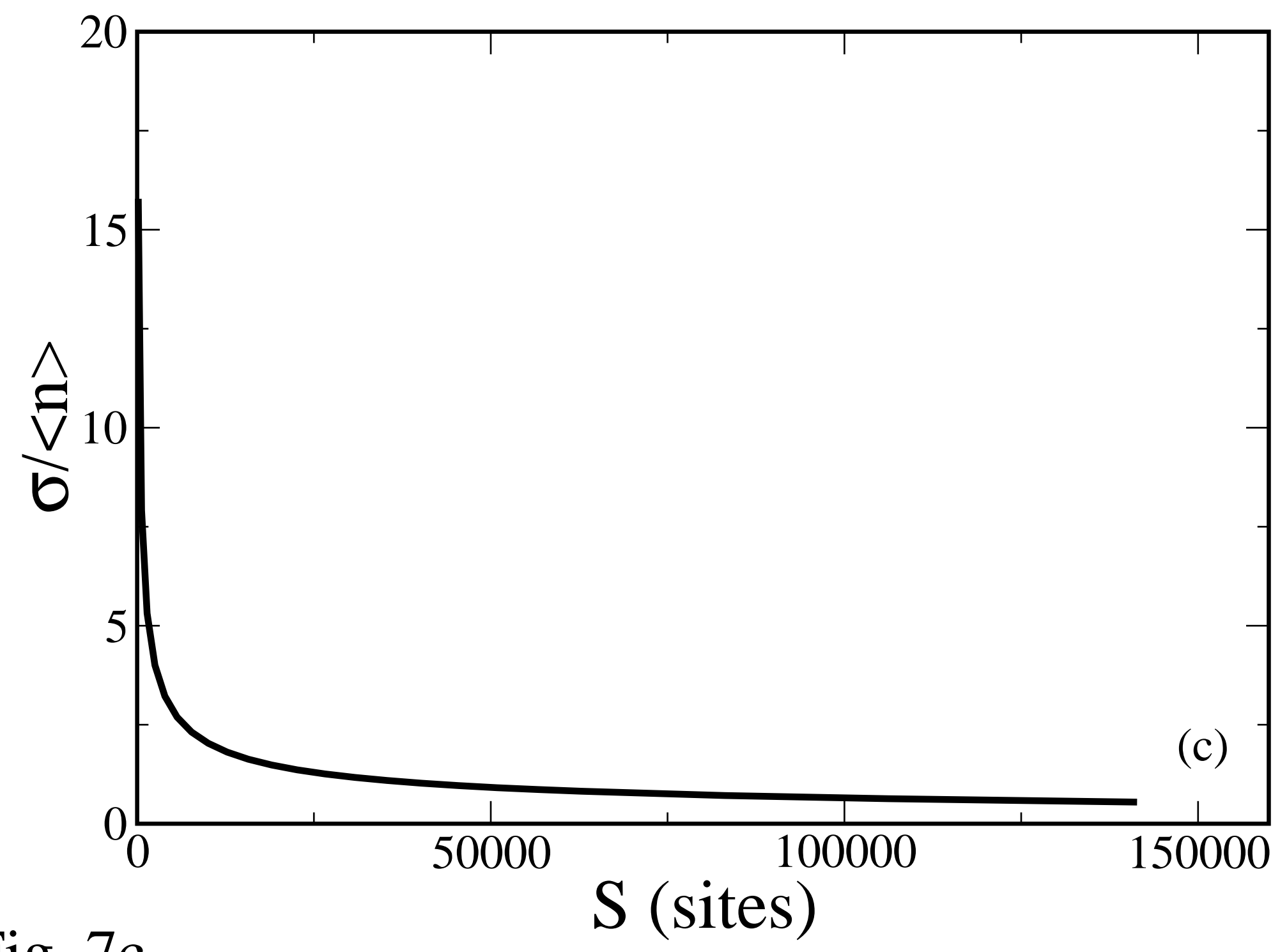

Fig. 7c 


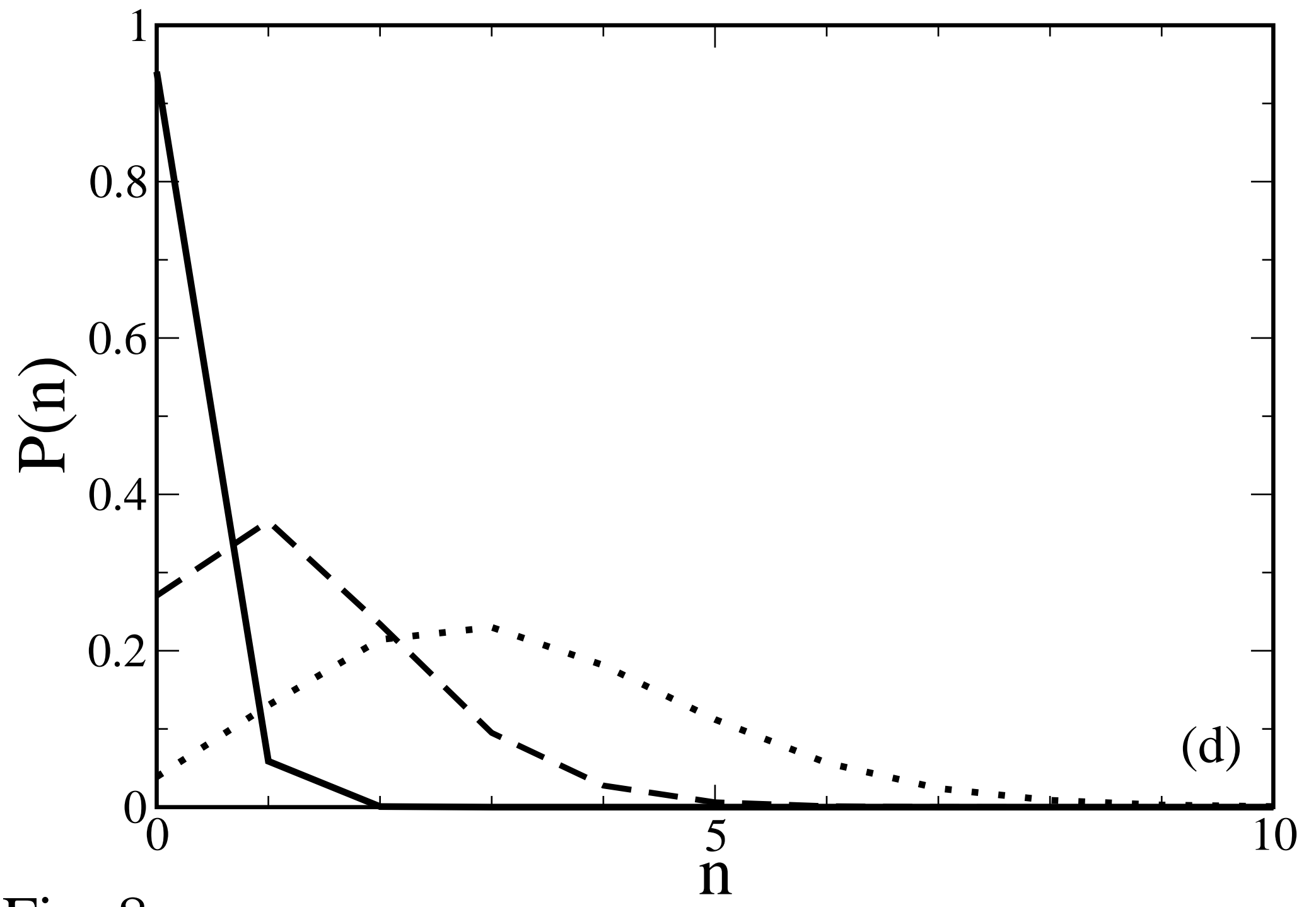

Fig. 8 